\title{
Infant Monitoring Using Old Smart Phones
}

\author{
Jonathan Fidelis Paul ${ }^{1}$, Tony Mathew ${ }^{2}$, R.Roseline Mary ${ }^{3}$ \\ 1,2,3 (Department of Computer Science, Christ University, Bengaluru , India
}

\begin{abstract}
The paper proposes a mobile app (InFi Care), which is an all-in-one infant monitoring system for parents who find it difficult to keep an eye on their infants while doing their daily chores. This work has been inspired by the motivation to reuse the old smart phones, which have numerous smart components ( sensors, like camera and microphone) that are not realizing their full potential, converting them into monitoring devices. The system works on a client server model in which a client monitors the environment for motion or a continuous vocal disturbance. On detecting any such activity of interest, the client notifies the server, situated on another smart phone, on the same network. The server is thus capable of being notified by several clients, enabling a centralized monitoring system using only old smartphones. InFi Care can be used in any smart phone with a camera and microphone with minimum 1GB RAM. It will help reduce e-waste, as people can reuse their old phones. This system has been designed to cater to the needs of parents with new born babies, who need erratic attention. A mother can be busy cooking in the kitchen while her baby is sleeping in a bassinet in some other room. A client phone will be setup to monitor the child, while the server phone will be with the mother. When the baby wakes up and starts moving or crying, the mother will be notified about this activity. The system can also be used in any place where an automation of noise or motion detection is required.
\end{abstract}

Keywords: Mobile app, realtime monitoring, baby care, image processing, sound processing

\section{Introduction}

A problem generally faced by nuclear families is that the parents tend to be busy monitoring their kids, ensuring they do not mess up the house, or cause any mischievous activities. This takes a good amount of their valuable time, which they regret for. Be it a sleeping kid or a toddler who is learning his new environment; all require persistent attention. A system which would monitor these activities and trigger an alarm on detecting abnormal activities would be of great help for parents. They could complete their regular chores as well as be carefree of their kids' whereabouts. In this project we have proposed a system which can monitor babies who are sleeping, children playing games or any activity in general, which may require monitoring. The system has a client server model. Since smart phones have become very common and having two or three phones in a house is normal, we decided to use smart phones to act as the client and server. With the added benefits of smart phones being equipped with sensors like cameras and gyroscope, we do not have to use any additional hardware. A number of apps (WiFi Camera Plus and Home Security Camera to name a few) are available which make use of the hardware support in android phones. These make use of the cloud services which will be unavailable if an internet connection is absent. More over these apps are cluttered with lots of options and are thus not domain specific, that is, catering to child monitoring. We have kept the system very simple in this project. One smart phone will start a server and the other will connect as a client to it. The server will capture a video from the scene and send it over WiFi to the client. In case of any activity the server sends a notification to the client phone. This is an alert to the parent to take action soon. This system thus gives the power in the hands of the parents to perform constant surveillance.

\section{Literature Review}

IP Webcam [1] turns a phone into a network camera with numerous viewing options. One can view their camera on any platform with a web browser or VLC player. Video streaming on a WiFi network is possible without internet access. There is an optional Ivideon cloud broadcasting support for instant global access. A two-way audio is supported in tinyCam Monitor on another android device. One can use IP Webcam with third-party MJPG software, including video surveillance software, security monitors and most audio players.

Wi-Fi Camera (Web of Cam) [2] allows one to monitor the surroundings with just one mobile phone, at anytime and from anywhere. By installing Web of Cam onto ones Android, iOS or Nokia phones or tablets, one will be able to see videos from another mobile phone or PC browser at real time via a Wi-Fi network. One can also configure their mobile phone into a Camera or Viewer unit. Alternatively, they can enter the URL displayed on the Camera device to a web browser and watch the video from PC. 
Sound Wire [3] lets one stream any music or audio from their Windows or Linux PC to their Android mobile devices. One can use it as:

- A remote speaker or wireless headphones

- A way to listen to music and movies from your computer anywhere around their house, or further over cell networks

- A wireless extension of live audio from your PC-based music system

Sound Wire does audio mirroring (audio cast). One can use any music player on your PC or laptop like Spotify, YouTube, or iTunes and stream the live sound directly to their Android device.
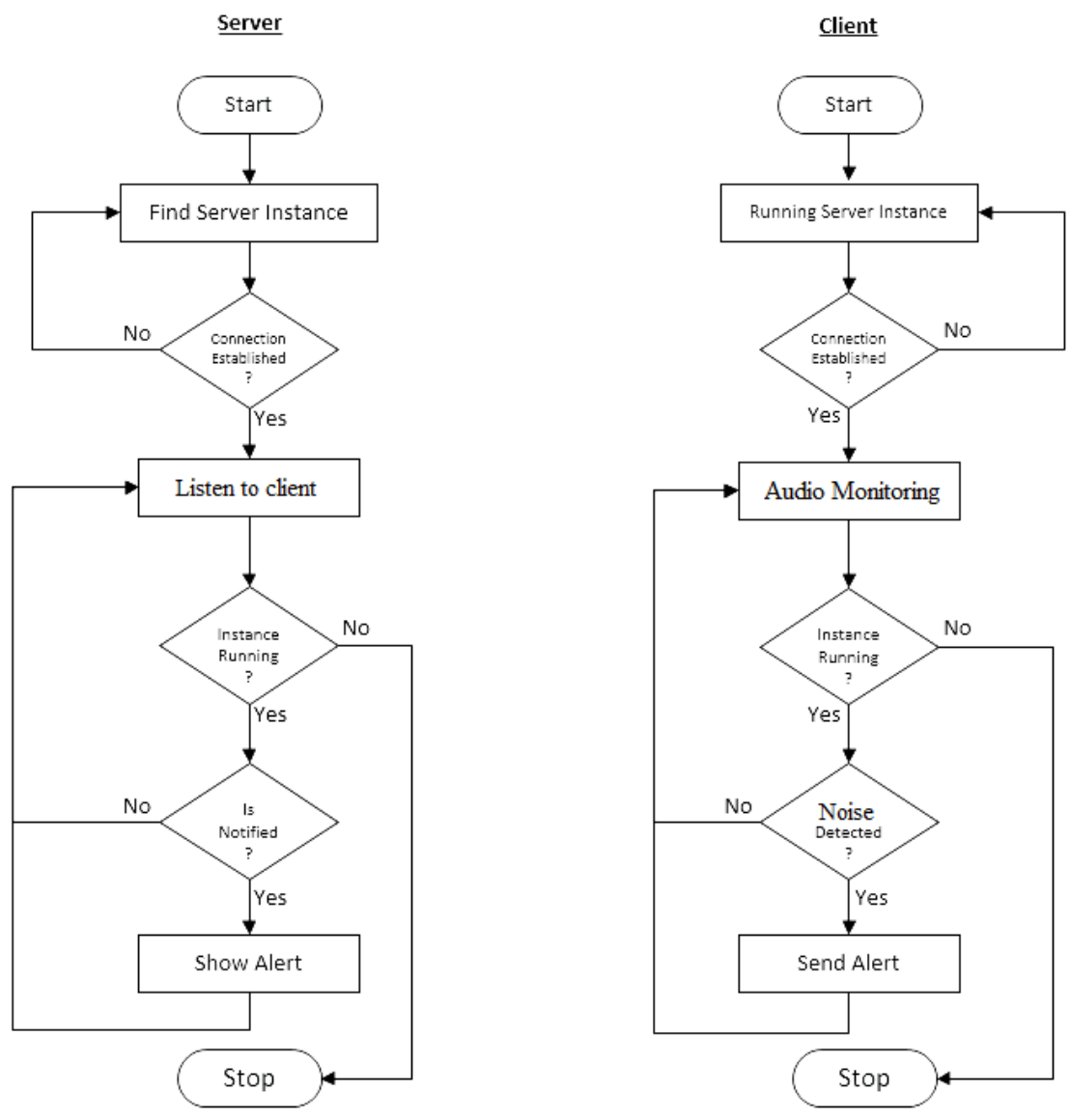

\section{Illustration 1: Flow Chart}

\section{Methodology}

In this paper a mobile application (InFi-Care) has been created to monitor an environment for noise and motion. Each step involved to do so had its own crucial role. This paper uses web sockets [10] to create a connection between two systems (android powered mobile phones). A server and a client are created on these mobile devices. The client is entrusted with the responsibility of monitoring a specific environment (figure 1). Numerous clients can be set up to monitor multiple rooms. Multiple clients can be answerable to a single server as in figure 2. This facilitates one server to have a check everywhere in the house.

\section{Implementation}

The mobile app was implemented in android. It has two modes: Client mode and Server Mode (as in figure 3)

\subsection{Client Mode}

It has all the monitoring features. The options available are sudden spike monitor and baby monitor.

The client is provided with audio monitoring features. While monitoring the client listens for high amplitudes of sound through the phone's microphone. A threshold is set by the user to be alerted if any sound is detected above it. On detecting a noticeable sound the client sends an alert message code to the dedicated server. At the server the message code is decoded and an automatic alarm notifies the parent. A screen shot of the present scene is also sent to the server, which pops up as a notification. The screen shot gives a clue to the parent about the reason for the alert. A backup of the latest alert is kept on the client. If an acknowledgment is not received from the server, the client re-sends the notification along with the screen shot.

DOI: $10.9790 / 0050-04020104 \quad$ www.iosrjournals.org $\quad 2 \mid$ Page




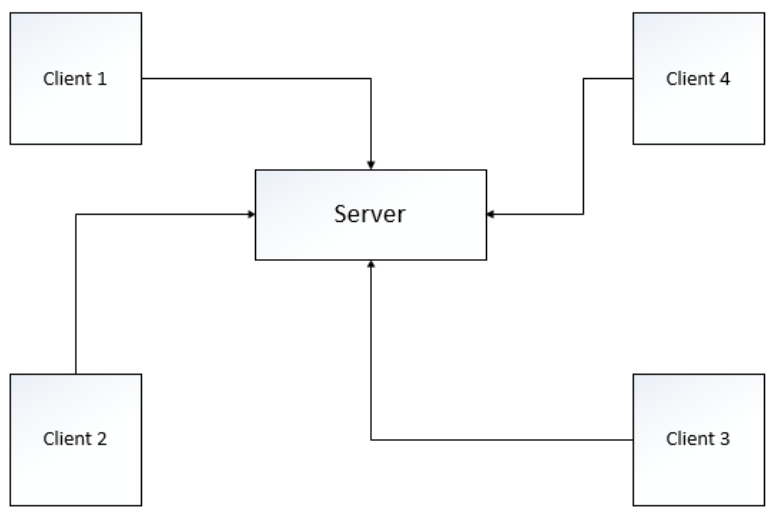

Illustration 2: Client Server Connection

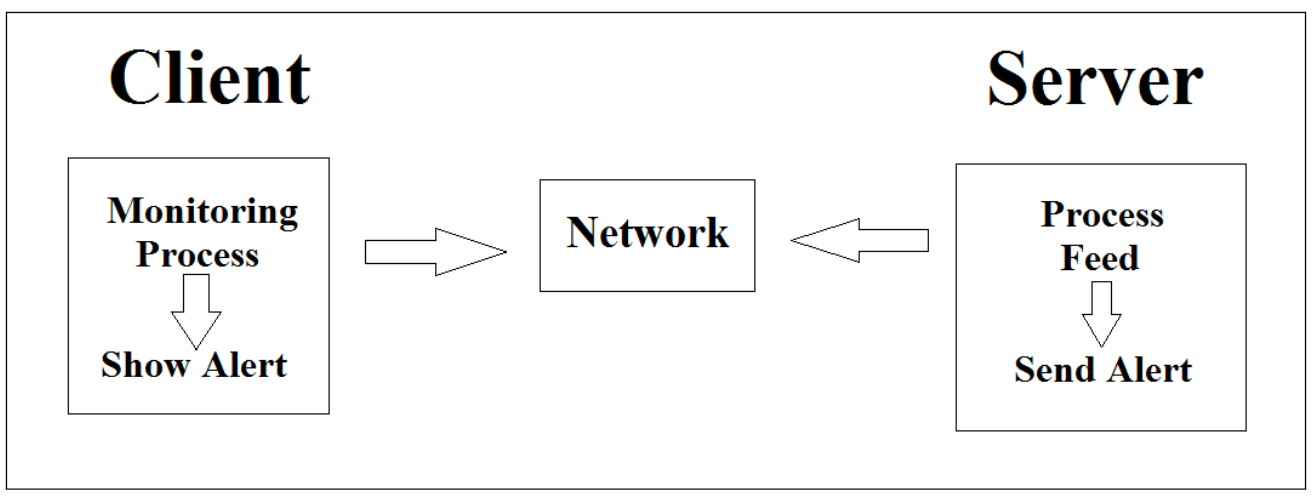

Illustration 3: System Architecture

\subsection{Server Mode}

It generates a service which can be detected by devices in client mode on the network. This network service discovery feature enables any client device to connect to a dedicated server to send monitored information.

The IP address of the server is shared to the clients and stored in their recent history. The clients can then use the same IP address to communicate with the server.

The server is always listening for client connections once it is started. On receiving any client request, it acts accordingly. In this paper the server and client communicate using message-codes. The codes used are CONNECT, RECORD, ALARM ON, ALARM OFF.

When the server receives any one of these codes it changes its state.

\section{Results}

The app has a neat interface, which enables quick and smooth use, for enhancing user experience. It can perform all actions within four clicks. The system works efficiently indoors. .

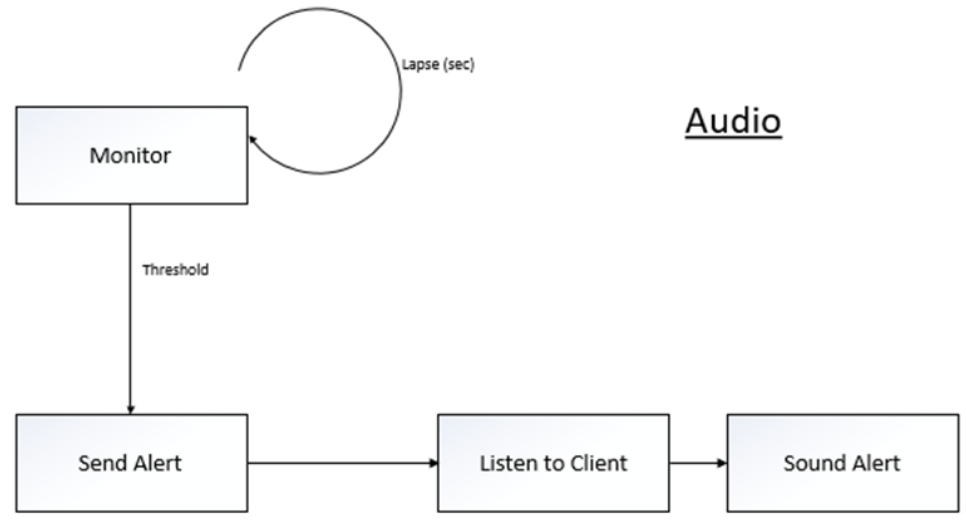

Illustration 4: Audio Monitoring Flow 


\section{Conclusion}

InFi-Care provides monitoring support which is charm when it comes to deal with a sleeping child. Since a child's cry is an indication that it is in need of some care, parents can use this system to monitor their children very efficiently. It provides smart monitoring, thus the two modes, sudden spikes of noise and baby cry monitor, have proven to be very useful. The system works best in an enclosed environment where noise is almost negligible.

\section{Future Enhancements}

To bring an improvement in this system we can add motion detection and face detection features which can monitor the movements in the surrounding environment and make smart decisions for alerting the parent. The notifications should also be smart with a snapshot of the environment or a 2 second video clip.

\section{References}

[1] Pavel Khlebovich, IP Webcam, 11th August 2016, https://play.google.com/store/apps/details?id=com.pas.webcam

[2] Alfred Labs Inc, Free Wi-Fi era (Web of Cam), 3rd February https://play.google.com/store/apps/details ?id=com.webofcam.

[3] GeorgieLabs, Sound Wire, 4th January $2016 \mathrm{https} / / /$ play.google.com/store/apps/details ?id=com.georgie.Sound Wire Free \&amp;hl=en

[4] Wikipedia, Android Studio, https://en.wikipedia.org/wiki/Android Studio

[5] Wikipedia,Android, https://en.wikipedia.org/wiki/Android (operating system)

[6] Wikipedia, Wireless https://en.wikipedia.org/wiki/Wireless LAN.

[7] Guru, 1st July 2015, androidsrc.net: client implementation, http://androidsrc.net/android-client-server- using-sockets-clientimplementation/

[8] Guru, 1st July 2015, androidsrc.net: server implementation, http://androidsrc.net/android-client-server-using-sockets- serverimplementation/

[9] Francis Dsa, 13th August 2013, Stream audio from PC to android [FirstPost/Tech],http://tech.firstpost.com/news-analysis/how-tostream-pc-audio-to-your-android-device-via-wi-fi-103912.html

[10] Android Developer Forum, Socket Program ming, https://developer.android.com/reference/java/net/Socket.html 